\title{
RANK-REVEALING QR FACTORIZATIONS AND THE SINGULAR VALUE DECOMPOSITION
}

\author{
Y. P. HONG AND C.-T. PAN
}

\begin{abstract}
T. Chan has noted that, even when the singular value decomposition of a matrix $A$ is known, it is still not obvious how to find a rank-revealing $\mathrm{QR}$ factorization (RRQR) of $A$ if $A$ has numerical rank deficiency. This paper offers a constructive proof of the existence of the RRQR factorization of any matrix $A$ of size $m \times n$ with numerical rank $r$. The bounds derived in this paper that guarantee the existence of $\mathrm{RRQR}$ are all of order $\sqrt{n r}$, in comparison with Chan's $O\left(2^{n-r}\right)$. It has been known for some time that if $A$ is only numerically rank-one deficient, then the column permutation $\Pi$ of $A$ that guarantees a small $r_{n n}$ in the QR factorization of $A \Pi$ can be obtained by inspecting the size of the elements of the right singular vector of $A$ corresponding to the smallest singular value of $A$. To some extent, our paper generalizes this well-known result.
\end{abstract}

\section{INTRODUCTION}

We consider the interplay between two important matrix decompositions: the singular value decomposition and the $\mathrm{QR}$ factorization of a matrix $A$. In particular, we are interested in the case when $A$ is singular or nearly singular. It is well known that for any $A \in \mathbb{R}^{m \times n}$ (a real matrix with $m$ rows and $n$ columns, where without loss of generality we assume $m \geq n$ ) there are orthogonal matrices $U$ and $V$ such that

$$
U^{\mathrm{T}} A V=\left[\begin{array}{l}
\Sigma \\
0
\end{array}\right],
$$

where $\Sigma$ is a diagonal matrix with nonnegative diagonal elements:

$$
\Sigma=\operatorname{diag}\left(\sigma_{1}, \sigma_{2}, \ldots, \sigma_{n}\right) .
$$

We assume that $\sigma_{1} \geq \sigma_{2} \geq \cdots \geq \sigma_{n} \geq 0$. The decomposition (0.1) is the singular value decomposition (SVD) of $A$, and the $\sigma_{i}$ are the singular values of $A$. The columns of $V$ are the right singular vectors of $A$, and the columns of $U$ are the left singular vectors of $A$. Mathematically, in terms of the singular values,

Received December 1, 1990; revised February 8, 1991.

1991 Mathematics Subject Classification. Primary 65F30, 15A23, 15A42, 15 A15.

Key words and phrases. Singular value decomposition, rank-revealing QR factorization, numerical rank, numerical null space.

Part of this work was performed while the second author was visiting Argonne National Laboratory under Argonne's Faculty Research Participation program, and was supported in part by the Applied Mathematical Sciences subprogram of the Office of Energy Research, U.S. Department of Energy, under Contract W-31-109-Eng-38. 
$A$ has rank $r$ (or nullity $(n-r)$ ) if and only if $\sigma_{r}>0$ and $\sigma_{r+1}=0$. However, computationally, when $\sigma_{r+1}$ is not exactly equal to zero but $\sigma_{r+1}=O(\mu)$, and if $\sigma_{r} \gg \sigma_{r+1}$, where $\mu$ is the machine precision and "》" means that there is an obvious magnitude gap between $\sigma_{r}$ and $\sigma_{r+1}$, we may say that $A$ has numerical rank $r$, or has numerical nullity $(n-r)$ (see Definition 1.1). In many applications, for a given matrix $A$, it is desirable to find the numerical rank $[8,21]$. Currently, the SVD is the most reliable, though expensive, numerical method for determining the numerical rank of a matrix.

An alternative, more practical method is $Q R$ factorization with column pivoting, a method proposed by Golub in the mid-sixties [9]. It is much cheaper than the SVD and useful in many applications such as solving rank-deficient least squares problems $[3,11]$. The method consists in using a column pivoting strategy [9] to determine a permutation matrix $\Pi$ such that $A \Pi=Q R$ is the QR factorization of $A \Pi$, with $Q \in \mathbb{R}^{m \times n}$ satisfying $Q^{T} Q=I_{n}$ and the upper triangular matrix $R$ partitioned as

$$
R=\left[\begin{array}{cc}
R_{11} & R_{12} \\
0 & R_{22}
\end{array}\right]
$$

where $R_{11} \in \mathbb{R}^{r \times r}$ and $R_{22}$ is small in norm (we hope). If, say, $\left\|R_{22}\right\|_{2}=O(\mu)$, then from the fact that $\sigma_{r+1} \leq\left\|R_{22}\right\|_{2}$ (see Lemma 1.2) we conclude that the original matrix $A$ is guaranteed to have at most numerical rank $r$.

The QR factorization of $A \Pi$, where $\Pi$ is a permutation matrix chosen to yield a "small" $R_{22}$, is referred to as the rank-revealing QR (RRQR) factorization of $A$ [6] (this is stated more precisely in Definition 1.3). While the RRQR factorization is another possible way to detect the numerical rank of a matrix, besides the SVD, the column pivoting strategy, unfortunately, does not always work. A well-known counterexample by Kahan [14, Example 3.1] shows that.

There are other strategies for finding an $R R Q R$ factorization of a matrix [2, $6,8,15,16,17,20]$, but most of them either simply fail to overcome Kahan's example, or provide no rigorous a priori bound for $\left\|R_{22}\right\|$. Chan [6] proposed an algorithm (similar ideas were independently proposed by Foster [8]) that does guarantee an RRQR factorization of $A$ if $A$ has low numerical nullity; in particular, Chan's algorithm works well for Kahan's example. It may work well in practice even when the numerical nullity is high, but not provenly so, since the bound derived in [6] is an exponential function of the numerical nullity.

Unlike other matrix decompositions that have been studied for centuries, there are still two fundamental unsolved questions that arise in the RRQR factorization. First, does there always exist a permutation matrix $\Pi$ such that $A \Pi=Q R$ is an $\mathrm{RRQR}$ factorization of $A$ if the numerical rank of $A$ is known? Second, if the permutation exists, how do we find it economically and reliably? Our paper gives an affirmative answer to the first question.

Our approach begins with the pioneering work of Golub, Klema, and Stewart [10]. Assume that the SVD of $A$ having numerical rank $r$ is known with $V=\left[V_{1}, V_{2}\right]$ in (0.1), where the columns of $V_{2} \in \mathbb{R}^{n \times(n-r)}$ are the right singular vectors corresponding to the smallest singular values $\sigma_{r+1}, \sigma_{r+2}, \ldots, \sigma_{n}$. It has been observed in [10] that the permutation matrix $\Pi$ which determines an RRQR factorization of $A$ is closely related to the selection of an $(n-r)$ by- $(n-r)$ submatrix of $V_{2}$ having a maximum smallest singular value among all submatrices of $V_{2}$ of the same size. However, the only proven result in 
this regard relates to the case when $A$ has numerical rank-one deficiency. In this case, $V_{2}$ is just a right singular vector $\mathbf{v}_{n}$ corresponding to the smallest singular value $\sigma_{n}$. It is then easy to show (see $\S 1$ ) that the permutation $\Pi$ such that $\left|\left(\Pi^{\mathrm{T}} \mathbf{v}_{n}\right)_{n}\right|=\left\|\mathbf{v}_{n}\right\|_{\infty}$ is the one that guarantees $\left|r_{n n}\right| \leq \sqrt{n} \sigma_{n}$, where $A \Pi=Q R$ is the $\mathrm{QR}$ factorization of $A \Pi$. When the numerical nullity of $A$ is greater than one, similar bounds on $\left\|R_{22}\right\|_{2}$ (or $\left\|R_{22}\right\|_{F}$ ), which guarantee an RRQR factorization of $A$, are not known. We shall establish such bounds in the general case.

It turns out that the selection of the submatrix with the maximum smallest singular value suggested in [10] can be replaced by the selection of a submatrix with maximum determinant. More precisely, if the SVD of $A$ is known (in particular, if the matrix $V_{2}$ is known), we show that the permutation matrix $\Pi=:\left[\Pi_{1}, \Pi_{2}\right]$ such that $\left|\operatorname{det}\left(\Pi_{2}^{\mathrm{T}} V_{2}\right)\right|$ is maximum among the absolute values of the determinants of all possible $(n-r)$-by- $(n-r)$ submatrices in $V_{2}$, guarantees an RRQR factorization of $A$. Nevertheless, our results are strictly theoretical; they offer no practical RRQR factorization algorithm since, if the SVD is known, there is no need to find an RRQR for revealing the numerical rank.

The rest of this paper is organized as follows. Section 1 gives basic notation and definitions as well as some preliminary results. Section 2 presents the main theorems. Section 3 illustrates the main theorem with numerical examples. In the final section, we state several remarks.

\section{Preliminaries}

1.1. Notation and basic concepts. In this section we define the notation used throughout this paper. We also define what we mean by the numerical rank and the RRQR factorization of a matrix $A$. We assume that all the matrices and vectors in this paper are real, but most results can be easily extended to complex numbers.

\section{Notation.}

- $\sigma_{i}(A)$, the singular values of the matrix $A$ in descending order,

- $\sigma_{\min }(A)$, the smallest singular value of $A$,

- $\mathscr{L}(W)$, the set of all $n$-by- $n$ submatrices of $W \in \mathbb{R}^{m \times n} \quad(m \geq n)$,

- $P_{n}$, the set of $n$-by- $n$ permutation matrices obtained by permuting the columns of the unit matrix $I_{n}$,

- $\mathscr{O}_{m, n}$, the set of $m$-by- $n$ matrices with orthonormal columns,

- $R_{11}, R_{12}, R_{22}$, the blocks of $R$, an upper triangular matrix, when $R$ is partitioned as $R=\left[\begin{array}{cc}R_{11} & R_{12} \\ 0 & R_{22}\end{array}\right]$,

- $G\left(i^{\prime}\right)$, a principal submatrix of a square matrix $G$ with the $i$ th row and $i$ th column of $G$ deleted,

- $\operatorname{det}(G)$, the determinant of a matrix $G$,

- $G \succ 0, G \succeq 0$, the matrix $G$ is positive definite, positive semidefinite, respectively,

- $(G)_{i j},(G)_{i:},(G)_{1: 5, j}$, the $(i, j)$ element, the $i$ th row, and the first five entries of the $j$ th column of a matrix $G$, respectively.

Bold lower-case letters denote vectors, $\|\cdot\|_{2}$ is the Euclidean norm of a vector or the spectral norm of a matrix, and $\|\cdot\|_{F}$ is the Frobenius norm of a matrix. 
Now we define what we mean by the numerical rank of a matrix.

Definition 1.1. A matrix $A \in \mathbb{R}^{m \times n}(m \geq n)$ is said to have numerical rank $r$ if

$$
\sigma_{r} \gg \sigma_{r+1}=O(\mu),
$$

where $\sigma_{1} \geq \sigma_{2} \geq \cdots \geq \sigma_{n} \geq 0$ are the singular values of $A$, and $\mu$ is the machine precision.

Here we use the notation " $a \gg b$ " to indicate that " $a$ is much larger than $b$." An alternative definition of numerical rank is given in $[8,10]$. For simplicity, we do not impose two constants to specify the "gap" between $a$ and $b$, as in [10]; but our definition is equivalent to the one called "numerical rank $(\delta, \varepsilon, r)_{2}$ " in [10] with $\sigma_{r} \geq \delta>\varepsilon \geq \sigma_{r+1}$, if the notation " $\gg$ " is specified by a pair of constants, $\delta$ and $\varepsilon$. We thus have two concepts of rank: the ordinary rank (which we sometimes call the exact rank), and the numerical rank. For simplicity, we do not give the full name of the rank when it is clear from the context which one we are referring to.

To define the RRQR factorization of a matrix $A$, we need the following lemma.

Lemma 1.2. If $A=Q R$ is the $Q R$ factorization of $A \in \mathbb{R}^{m \times n} \quad(m \geq n)$, with $R=\left[\begin{array}{cc}R_{11} & R_{12} \\ 0 & R_{22}\end{array}\right]$ and $R_{11} \in \mathbb{R}^{r \times r}$, and if

$$
\sigma_{\min }\left(R_{11}\right) \gg\left\|R_{22}\right\|_{2}=O(\mu),
$$

then $A$ has numerical rank $r$.

Proof. Let $\sigma_{1} \geq \cdots \geq \sigma_{n}$ be the singular values of $A$. From the singular value interlacing property [13, p. 417], it is easy to show that

$$
\sigma_{r} \geq \sigma_{\min }\left(R_{11}\right)
$$

and

$$
\left\|R_{22}\right\|_{2} \geq \sigma_{r+1} .
$$

Thus,

$$
\sigma_{r} \geq \sigma_{\min }\left(R_{11}\right) \gg\left\|R_{22}\right\|_{2} \geq \sigma_{r+1}=O(\mu),
$$

and the lemma is proved.

Because of Lemma 1.2, the following definition of an RRQR factorization of a matrix $A$ is justified (see also Remark 1 below).

Definition 1.3. Assume that a matrix $A \in \mathbb{R}^{m \times n} \quad(m \geq n)$ has numerical rank $r(<n)$. If there exists a permutation $\Pi \in P_{n}$ such that $A \Pi$ has a $\mathrm{QR}$ factorization $A \Pi=Q R$ with $R=\left[\begin{array}{cc}R_{11} & R_{12} \\ 0 & R_{22}\end{array}\right], R_{11} \in \mathbb{R}^{r \times r}$, and

$$
\sigma_{\min }\left(R_{11}\right) \gg\left\|R_{22}\right\|_{2}=O(\mu),
$$

then the factorizaton $A \Pi=Q R$ is called a Rank-Revealing $Q R$ factorization (RRQR) of $A$.

This definition poses conditions on both blocks $R_{11}$ and $R_{22}$. This is consistent with Definition 1.1. Once the $\Pi$ is found such that the corresponding $R$ satisfies (1.5), the numerical rank of $A$ is revealed. 
By definition, for given $A$ and $r$, the question of whether a QR factorization of $A \Pi$ is an RRQR factorization is decided solely by the values of $\sigma_{\min }\left(R_{11}\right)$ and $\left\|R_{22}\right\|_{2}$ corresponding to the $\Pi$ chosen. We know that the $\mathrm{QR}$ factorization of $A$ (or $A \Pi$ ) is unique (up to the signs of rows of $R$ ) when $A$ (or $A \Pi$ ) has full rank. In this case there is only one pair of $\sigma_{\min }\left(R_{11}\right)$ and $\left\|R_{22}\right\|_{2}$ for each $\Pi$ chosen. When $A$ is singular (exact rank-deficient), however, the QR factorization of $A \Pi$ is no longer unique in general. In this case, does it still make sense to use the values of $\sigma_{\min }\left(R_{11}\right)$ and $\left\|R_{22}\right\|_{2}$ to define the RRQR factorization of $A$ ? Will different $\Pi$ generate different pairs of $\sigma_{\min }\left(R_{11}\right)$ and $\left\|R_{22}\right\|_{2}$ (and if so, how many different pairs of $\sigma_{\min }\left(R_{11}\right)$ and $\left\|R_{22}\right\|_{2}$ are there for fixed $A$ and $r$ )? The following two remarks answer these questions.

Remark 1. For fixed $A, r$, and $\Pi$, if $\sigma_{\min }\left(R_{11}\right) \neq 0$, the values of $\sigma_{\min }\left(R_{11}\right)$ and $\left\|R_{22}\right\|_{2}$ are uniquely determined by the $\mathrm{QR}$ factorization of $A \Pi$ even though the $\mathrm{QR}$ factorization may not be unique.

Proof. Assume $A$ is a singular matrix. Suppose that

$$
A \Pi=Q\left(\begin{array}{cc}
R_{11} & R_{12} \\
0 & R_{22}
\end{array}\right)=\bar{Q}\left(\begin{array}{cc}
\bar{R}_{11} & \bar{R}_{12} \\
0 & \bar{R}_{22}
\end{array}\right)
$$

are two different QR factorizations of $A \Pi$. Then

$$
\begin{aligned}
& \left(\begin{array}{lc}
R_{11}^{\mathrm{T}} R_{11} & R_{11}^{\mathrm{T}} R_{12} \\
R_{12}^{\mathrm{T}} R_{11} & R_{12}^{\mathrm{T}} R_{12}+R_{22}^{\mathrm{T}} R_{22}
\end{array}\right) \\
& \quad=\left(\begin{array}{ll}
\bar{R}_{11}^{\mathrm{T}} \bar{R}_{11} & \bar{R}_{11}^{\mathrm{T}} \bar{R}_{12} \\
\bar{R}_{12}^{\mathrm{T}} \bar{R}_{11} & \bar{R}_{12}^{\mathrm{T}} \bar{R}_{12}+\bar{R}_{22}^{\mathrm{T}} \bar{R}_{22}
\end{array}\right)=\Pi^{\mathrm{T}} A^{\mathrm{T}} A \Pi .
\end{aligned}
$$

Clearly, $\sigma_{\min }\left(R_{11}\right)=\sigma_{\min }\left(\bar{R}_{11}\right)$. Since $R_{11}^{\mathrm{T}} R_{11}=\bar{R}_{11}^{\mathrm{T}} \bar{R}_{11} \succ 0$, the upper triangular matrices $R_{11}$ and $\bar{R}_{11}$ are equal up to the signs of rows. Now, comparing the $(1,1)$ - and $(1,2)$-blocks of $(1.6)$, and using the nonsingularity of $R_{11}$ and $\bar{R}_{11}$, we obtain $\bar{R}_{12}^{\mathrm{T}} \bar{R}_{12}=R_{12}^{\mathrm{T}} R_{12}$. Comparing the $(2,2)$-blocks in (1.6) then gives $R_{22}^{\mathrm{T}} R_{22}=\bar{R}_{22}^{\mathrm{T}} \bar{R}_{22}$, hence $\left\|R_{22}\right\|_{2}=\left\|\bar{R}_{22}\right\|_{2}$.

Remark 2. Let $A=Q\left[\begin{array}{cc}R_{11} & R_{12} \\ 0 & R_{22}\end{array}\right]$ be a QR factorization of $A$ with $\sigma_{\min }\left(R_{11}\right) \neq 0$. Suppose that $\Pi=\left[\begin{array}{cc}\Pi_{1} & 0 \\ 0 & \Pi_{2}\end{array}\right] \in P_{n}$ with $\Pi_{1} \in P_{r}$ and $\Pi_{2} \in P_{n-r}$. If

$$
A \Pi=\bar{Q}\left[\begin{array}{cc}
\bar{R}_{11} & \bar{R}_{12} \\
0 & \bar{R}_{22}
\end{array}\right]
$$

is any QR factorization of $A \Pi$, then $\sigma_{\min }\left(R_{11}\right)=\sigma_{\min }\left(\bar{R}_{11}\right)$ and $\left\|R_{22}\right\|_{2}=$ $\left\|\bar{R}_{22}\right\|_{2}$.

Proof. For

$$
A \Pi=Q\left[\begin{array}{cc}
R_{11} \Pi_{1} & R_{12} \Pi_{2} \\
0 & R_{22} \Pi_{2}
\end{array}\right],
$$

there exists an orthogonal matrix $Q^{\prime}=\left[\begin{array}{cc}Q_{1}^{\prime} & 0 \\ 0 & Q_{2}^{\prime}\end{array}\right]$ such that

$$
A \Pi=Q Q^{\prime}\left[\begin{array}{cc}
R_{11}^{\prime} & R_{12}^{\prime} \\
0 & R_{22}^{\prime}
\end{array}\right]
$$


is a $\mathrm{QR}$ factorization of $A \Pi$, where $\left(Q_{1}^{\prime}\right)^{\mathrm{T}} R_{11} \Pi_{1}=R_{11}^{\prime}$ and $\left(Q_{2}^{\prime}\right)^{\mathrm{T}} R_{22} \Pi_{2}=$ $R_{22}^{\prime}$. By Remark 1 the conclusion of this remark is now obvious.

Notice that the restriction $\sigma_{\min }\left(R_{11}\right) \neq 0$ in Remark 1 does not conflict with Definition 1.3 since, when $\sigma_{\min }\left(R_{11}\right)=0$, the corresponding QR factorization cannot be an RRQR one. From Remark 2, for fixed $A$ and $r$, if $\sigma_{\min }\left(R_{11}\right) \neq 0$ for all $\Pi \in P_{n}$, then there exist at most $C(n, r)=n ! /(n-r) ! r !$ distinct pairs of values of $\sigma_{\min }\left(R_{11}\right)$ and $\left\|R_{22}\right\|_{2}$. If for some $\Pi, \sigma_{\min }\left(R_{11}\right)=0$, it does not matter which $\left\|R_{22}\right\|_{2}$ we choose, since the corresponding $Q R$ is not an RRQR. Therefore, according to Remark 2, the maximum number of different QR factorizations needed to obtain an RRQR of $A$ (if it exists) is $C(n, r)$ (not $n$ !) if we know the numerical rank of $A$ is $r$. This is consistent with the fact that there are only $C(n, r)$ different $r$-by- $r$ submatrices in $V_{2}$ when we inspect either their smallest singular values or absolute determinant values to obtain a permutation that guarantees an RRQR of $A$. This fact is also quite useful when we try (cf. $\S 3$ ) to exhibit all possible $Q R$ factorizations of a given matrix for different permutation $\Pi$. (Remark 2 also justifies the suggestion of using so-called cyclic permutations [6] when one wishes to keep the original sparsity structure in seeking an RRQR.)

A final note on Definition 1.3 concerns the matrix norm used in the definition. Since all finite-dimensional matrix norms are equivalent, it is not necessary to use the 2-norm here. Often more useful is the Frobenius norm. Noting that $\|M\|_{2} \leq\|M\|_{F}$ for any matrix $M$, one can easily see that the following definition is an alternative to Definition 1.3.

Definition 1.4. Assume that a matrix $A \in \mathbb{R}^{m \times n} \quad(m \geq n)$ has numerical rank $r(<n)$. If there exists a permutation matrix $\Pi \in P_{n}$ such that $A \Pi$ has a $\mathrm{QR}$ factorization $A \Pi=Q R$ with $R=\left[\begin{array}{cc}R_{11} & R_{12} \\ 0 & R_{22}\end{array}\right], R_{11} \in \mathbb{R}^{r \times r}$, and

$$
\frac{1}{\left\|R_{11}^{-1}\right\|_{F}} \gg\left\|R_{22}\right\|_{F}=O(\mu) \text {, }
$$

then the factorization $A \Pi=Q R$ is called a Rank-Revealing $Q R$ factorization (RRQR) of $A$.

Lemma 1.2 shows that a gap between $\sigma_{\min }\left(R_{11}\right)$ and $\left\|R_{22}\right\|_{2}$ implies a gap between $\sigma_{r}$ and $\sigma_{r+1}$. In this paper, our main goal is to show the converse, i.e., a gap between $\sigma_{r}$ and $\sigma_{r+1}$ implies a gap between $\sigma_{\min }\left(R_{11}\right)$ and $\left\|R_{22}\right\|_{2}$.

1.2. Preliminary results. It has been noticed [10] that the permutation $\Pi$ that may lead to an RRQR factorization of $A$ is closely related to a certain selection of a subset of rows of a matrix whose columns are the right singular vectors corresponding to all near-zero singular values of $A$ : one chooses a number of rows equal to the number of near-zero singular values in such a way that the resulting square matrix has maximal smallest singular value. The following theorem explains why and how the permutation and the smallest singular value of a selected subset of the rows are related. We note that the inequality (1.8) has appeared in [10] in a slightly different form.

Theorem 1.5. For any matrix $A \in \mathbb{R}^{m \times n}(m \geq n)$, suppose that $A \Pi=Q R$ is the $Q R$ factorization of $A \Pi$ with some $\Pi \in P_{n}$. For any positive integer $r$ 
$(<n)$, let $R=\left[\begin{array}{cc}R_{11} & R_{12} \\ 0 & R_{22}\end{array}\right]$ be a partition of $R$, where $R_{11} \in \mathbb{R}^{r \times r}$. Then the following inequalities hold:

$$
\begin{gathered}
\sigma_{\min }\left(R_{11}\right) \geq \sigma_{\min }\left(\Pi_{1}^{\mathrm{T}} V_{1}\right) \cdot \sigma_{r}(A), \\
\left\|R_{22}\right\|_{2} \leq \frac{1}{\sigma_{\min }\left(\Pi_{2}^{\mathrm{T}} V_{2}\right)} \cdot \sigma_{r+1}(A) .
\end{gathered}
$$

It is assumed here that $\Pi=\left[\Pi_{1}, \Pi_{2}\right]$ and $V=\left[V_{1}, V_{2}\right]$ with $\Pi_{1}, V_{1} \in \mathbb{R}^{n \times r}$, where $U^{\mathrm{T}} A V=\Sigma$ is the SVD of $A$. Moreover, there holds

$$
\sigma_{\min }\left(\Pi_{1}^{\mathrm{T}} V_{1}\right)=\sigma_{\min }\left(\Pi_{2}^{\mathrm{T}} V_{2}\right) .
$$

Proof. Let $\left[\widehat{U}_{1}, \widehat{U}_{2}\right]=\widehat{U}=U \Sigma$, where $\widehat{U}_{1} \in \mathbb{R}^{m \times r}$. Thus $A V=\widehat{U}$ or $A=$ $\widehat{U}_{1} V_{1}^{\mathrm{T}}+\widehat{U}_{2} V_{2}^{\mathrm{T}}$. It is easy to check that

$$
\begin{aligned}
\sigma_{\min }\left(A \Pi_{1}\right) & =\sigma_{\min }\left(\widehat{U}_{1} V_{1}^{\mathrm{T}} \Pi_{1}+\widehat{U}_{2} V_{2}^{\mathrm{T}} \Pi_{1}\right) \\
& \geq \sigma_{\min }\left(\widehat{U}_{1} V_{1}^{\mathrm{T}} \Pi_{1}\right) \geq \sigma_{\min }\left(\widehat{U}_{1}\right) \cdot \sigma_{\min }\left(\Pi_{1}^{\mathrm{T}} V_{1}\right) .
\end{aligned}
$$

On the other hand, $\sigma_{\min }\left(A \Pi_{1}\right)=\sigma_{\min }\left(Q\left[\begin{array}{c}R_{11} \\ 0\end{array}\right]\right)=\sigma_{\min }\left(R_{11}\right)$, and $\sigma_{\min }\left(\widehat{U}_{1}\right)=$ $\sigma_{r}(A)$. Thus, inequality (1.8) is proved.

Observe that

$$
Q R \Pi^{\mathrm{T}} V_{2}=A \Pi \Pi^{\mathrm{T}} V_{2}=A V_{2}=\widehat{U}_{2} .
$$

It is easy to check that

$$
\sigma_{r+1}(A)=\left\|\widehat{U}_{2}\right\|_{2}=\left\|R \Pi^{\mathrm{T}} V_{2}\right\|_{2} \geq\left\|R_{22} \Pi_{2}^{\mathrm{T}} V_{2}\right\|_{2} \geq\left\|R_{22}\right\|_{2} \cdot \sigma_{\min }\left(\Pi_{2}^{\mathrm{T}} V_{2}\right) .
$$

Thus, inequality (1.9) is also true. Now notice that the orthogonal matrix $\Pi^{\mathrm{T}} V$ can be partitioned as

$$
\Pi^{\mathrm{T}} V=\left[\begin{array}{ll}
\Pi_{1}^{\mathrm{T}} V_{1} & \Pi_{1}^{\mathrm{T}} V_{2} \\
\Pi_{2}^{\mathrm{T}} V_{1} & \Pi_{2}^{\mathrm{T}} V_{2}
\end{array}\right]
$$

According to the C-S decomposition theorem [11, p. 77], equation (1.10) is immediate.

Inequalities (1.8) and (1.9) may now be written as

$$
\sigma_{\min }\left(R_{11}\right) \geq \rho \sigma_{r}(A)
$$

and

$$
\left\|R_{22}\right\|_{2} \leq \frac{1}{\rho} \sigma_{r+1}(A),
$$

where $\rho=\sigma_{\min }\left(\Pi_{1}^{\mathrm{T}} V_{1}\right)=\sigma_{\min }\left(\Pi_{2}^{\mathrm{T}} V_{2}\right)$.

Remark 3. In Theorem 1.5, if $V$ is replaced by $V\left[\begin{array}{cc}Z_{1} & 0 \\ 0 & Z_{2}\end{array}\right]$, where $Z_{1} \in \mathbb{R}^{r \times r}$ and $Z_{2} \in \mathbb{R}^{(n-r) \times(n-r)}$ are orthogonal matrices, one can easily verify that the corresponding inequalities still hold. In particular, if $V_{2}$ is replaced by any orthonormal basis of the column space of $V_{2}$, the numerical null space of $A$, Theorem 1.5 is still valid.

Theorem 1.5 is important since it provides us with a mechanism for identifying the possible candidates of $\Pi$ which may guarantee an RRQR factorization of $A$. To explain this, we introduce some additional notation. Let $W \in \mathscr{O}_{n, r}$ with $n \geq r$, and let $\mathscr{L}(W)$ be the set of all $r$-by-r submatrices of $W$. If we 
order these submatrices in some way (for example, using lexicographical order according to the row indices in the original matrix $W$ ) as $W_{1}, W_{2}, \ldots, W_{p}$, where $p=C(n, r)$, we can define a vector in $\mathbb{R}^{p}$ by

$$
\boldsymbol{\sigma}(W)=\left(\sigma_{\min }\left(W_{1}\right), \sigma_{\min }\left(W_{2}\right), \ldots, \sigma_{\min }\left(W_{p}\right)\right)^{\mathrm{T}} .
$$

For all $W \in \mathscr{O}_{n, r}$, if one could show that $\inf _{W \in \mathcal{O}_{n, r}}\left(\|\boldsymbol{\sigma}(W)\|_{\infty}\right)$ is a number not too small, say $O(\sqrt{1 / n})$, then according to Theorem 1.5 the existence of an RRQR factorization is guaranteed by choosing a $\widehat{\Pi} \in P_{n}$ such that $\sigma_{\min }\left(\widehat{\Pi}_{1}^{\mathrm{T}} V_{1}\right)$ is maximum among all possible $r$-by- $r$ submatrices of $V_{1}$ (at the same time $\sigma_{\min }\left(\widehat{\Pi}_{2}^{\mathrm{T}} V_{2}\right)$ is maximum among all possible $(n-r)$-by- $(n-r)$ submatrices of $\left.V_{2}\right)$, since then

$$
\rho=\sigma_{\min }\left(\widehat{\Pi}_{1}^{\mathrm{T}} V_{1}\right)=\sigma_{\min }\left(\widehat{\Pi}_{2}^{\mathrm{T}} V_{2}\right) \geq \inf _{W \in \mathscr{O}_{n, r}}\left(\|\boldsymbol{\sigma}(W)\|_{\infty}\right) .
$$

Note that we need to consider only $C(n, r)$ elements in $P_{n}$ as candidates of $\widehat{\Pi}$, since permuting the rows of a matrix will not change the singular values of the matrix. This fact is consistent with Remark 2 . We define the following function of $n$ and $r$ :

$$
\chi(n, r)=: \inf _{W \in \mathscr{Q}_{n, r}}\left(\|\boldsymbol{\sigma}(W)\|_{\infty}\right) .
$$

From the C-S decomposition theorem one easily sees that

$$
\chi(n, r)=\chi(n, n-r) \text {. }
$$

Thus, the existence of a permutation, which guarantees an RRQR factorization for an arbitrary matrix $A$ with numerical rank $r$, depends entirely on the value of $\chi(n, r)$. Therefore, the rest of this paper is devoted to obtaining the value of $\chi(n, r)$, or a sufficiently sharp lower bound for it. When $r=1$ or $r=n-1$, this is trivial.

Lemma 1.6. Let $\mathbf{v} \in \mathbb{R}^{n}$ and $\|\mathbf{v}\|_{2}=1$. Then

$$
\chi(n, n-1)=\chi(n, 1)=\inf _{\|\mathbf{v}\|_{2}=1}\left(\|\mathbf{v}\|_{\infty}\right)=1 / \sqrt{n} .
$$

Proof. It is clear that $\|\mathbf{v}\|_{\infty} \geq 1 / \sqrt{n}$ for each $\mathbf{v} \in \mathbb{R}^{n}$ with $\|\mathbf{v}\|_{2}=1$, since otherwise $v_{i}<1 / \sqrt{n}$ for each $i$, giving $\|\mathbf{v}\|_{2}<1$. On the other hand, the vector $(1 / \sqrt{n}, \ldots, 1 / \sqrt{n})^{\mathrm{T}}$ achieves the lower bound $1 / \sqrt{n}$.

From this lemma and Theorem 1.5 we immediately have the following corollary for the rank-one deficient case $(r=n-1)$.

Corollary 1.7. For any matrix $A \in \mathbb{R}^{m \times n} \quad(m \geq n)$, there exists a permutation $\Pi \in P_{n}$ such that $A \Pi$ has a $Q R$ factorization $A \Pi=Q R$ with

$$
\sigma_{\min }\left(R_{11}\right) \geq \frac{1}{\sqrt{n}} \sigma_{n-1}(A) \text { and }\left|r_{n n}\right| \leq \sqrt{n} \sigma_{n}(A) \text {, }
$$

where $R_{11} \in \mathbb{R}^{(n-1) \times(n-1)}$. If $\mathbf{v}_{n}$ is the right singular vector associated with $\sigma_{n}(A)$, the permutation $\Pi$ is given by $\left|\left(\Pi^{\mathrm{T}} \mathbf{v}_{n}\right)_{n}\right|=\left\|\mathbf{v}_{n}\right\|_{\infty}$. Moreover, if $A$ is numerically rank-one deficient, the $Q R$ factorization of $A \Pi$ is guaranteed to be an $R R Q R$ of $A$.

Corollary 1.7 was first pointed out in [10]. It is also the backbone of Chan's algorithm [6]. However, even for the rank-two deficient case, not to mention 
the general case, much more effort is required to obtain the value of $\chi(n, r)$ or a sufficiently sharp lower bound for it. The following result addresses the rank-two deficient case, but its lengthy proof is deferred to the Appendix.

Lemma 1.8. Let $V \in \mathscr{O}_{n, 2}$. Then

$$
\begin{aligned}
\chi(n, n-2) & =\chi(n, 2)=\inf _{V \in \mathscr{O}_{n, 2}}\left(\|\boldsymbol{\sigma}(V)\|_{\infty}\right) \\
& \geq \sqrt{(2-\sqrt{2} \sqrt{(n-2) /(n-1)}) / n} .
\end{aligned}
$$

Correspondingly, we have the following corollary.

Corollary 1.9. For any matrix $A \in \mathbb{R}^{m \times n} \quad(m \geq n)$, there exists $a \Pi \in P_{n}$ such that $A \Pi$ has a $Q R$ factorization $A \Pi=Q R$ with

$$
\sigma_{\min }\left(R_{11}\right) \geq \sqrt{(2-\sqrt{2} \sqrt{(n-2) /(n-1)}) / n} \cdot \sigma_{n-2}(A)
$$

and

$$
\left\|R_{22}\right\|_{2} \leq \frac{1}{\sqrt{(2-\sqrt{2} \sqrt{(n-2) /(n-1)}) / n}} \cdot \sigma_{n-1}(A),
$$

where $R_{11} \in \mathbb{R}^{(n-2) \times(n-2)}$. If $V_{2}=\left[\mathbf{v}_{n-1}, \mathbf{v}_{n}\right]$, where $\mathbf{v}_{n-1}$ and $\mathbf{v}_{n}$ are the right singular vectors associated with $\sigma_{n-1}(A)$ and $\sigma_{n}(A)$, respectively, the permutation matrix $\Pi$ is given by $\sigma_{\min }\left(\Pi^{\mathrm{T}} V_{2}\right)_{n-1: n, n-1: n}=\left\|\sigma\left(V_{2}\right)\right\|_{\infty}$. Moreover, when $A$ is numerically rank-two deficient, the $Q R$ factorization of $A \Pi$ is an $R R Q R$ of $A$.

The proof of Lemma 1.8 exhibits the difficulty of obtaining sharp lower bounds for the function $\chi(n, r)$ directly, even when $r=2$. In the next section, we will use a different approach to prove the existence of an RRQR factorization of a matrix having any numerical rank.

\section{EXISTENCE THEOREMS FOR RRQR FACTORIZATION}

By Theorem 1.5, the existence of an RRQR seems to be closely related to the smallest singular values of the submatrices of a matrix whose columns form an orthonormal basis for the numerical null space of $A$. In this section, we show that the determinants of these submatrices are alternative parameters which allow us to identify the permutation matrix that guarantees an RRQR. In fact, we show that the submatrix having the largest determinant in absolute value also has a large enough smallest singular value, provided the submatrix is chosen from a matrix with orthonormal columns. The following lemma will lead to our main theorem.

Lemma 2.1. Let $V \in \mathscr{O}_{n, r}(n>r)$. If

$$
\widehat{\Pi}=\left[\begin{array}{l}
\widehat{\Pi}_{1} \\
\widehat{\Pi}_{2}
\end{array}\right] \in P_{n} \quad\left(\widehat{\Pi}_{2} \in \mathbb{R}^{r \times n}\right)
$$

is a permutation matrix such that $\left|\operatorname{det}\left(\widehat{\Pi}_{2} V\right)\right|=\max _{V^{\prime} \in \mathscr{L}(V)}\left|\operatorname{det}\left(V^{\prime}\right)\right|$, then the following inequality holds:

$$
\max _{1 \leq i \leq r} \frac{\operatorname{det} G\left(i^{\prime}\right)}{\operatorname{det} G} \leq n-r+1,
$$

where $G=\left(\widehat{\Pi}_{2} V\right)\left(\widehat{\Pi}_{2} V\right)^{\mathrm{T}}$. 
Proof. Let $\mathscr{L}(V)=\left\{V_{1}, V_{2}, \ldots, V_{p}\right\}$, where $p=C(n, r)=n ! /(n-r) ! r !$, be the set of all different $r$-by-r submatrices of $V$. Since $\operatorname{rank}(V)=r$, there exists a nonsingular matrix $V_{*} \in \mathscr{L}(V)$ such that $\left|\operatorname{det}\left(V_{*}\right)\right|=\max _{1 \leq i \leq p}\left|\operatorname{det}\left(V_{i}\right)\right| \neq 0$. Consequently, there must exist a permutation $\widehat{\Pi}=\left[\begin{array}{l}\widehat{\Pi}_{1} \\ \widehat{\Pi}_{2}\end{array}\right]$ such that $\widehat{\Pi}_{2} V=V_{*}$. Let $\widehat{\Pi} V=: \widehat{V}$, and $Q \in \mathbb{R}^{r \times r}$ be an orthogonal matrix such that

$$
\widehat{V} Q=\left[\begin{array}{c}
\widetilde{V}_{[1]} \\
\widetilde{V}_{[2]}
\end{array}\right]=: \widetilde{V}
$$

and $\widetilde{V}_{[2]}$ is an upper triangular matrix.

From $\widetilde{V}^{\mathrm{T}} \widetilde{V}=Q^{\mathrm{T}} V^{\mathrm{T}} \hat{\Pi}^{\mathrm{T}} \hat{\Pi} V Q=I_{r}$, which is the $r$-by-r unit matrix, we know that $\widetilde{V} \in \mathscr{O}_{n, r}$. In particular, the 2-norm of the vector

$$
(\tilde{V}): 1=\left(\begin{array}{c}
s_{1} \\
s_{2} \\
\vdots \\
s_{n-r+1} \\
0 \\
\vdots \\
0
\end{array}\right)=: \mathbf{s}
$$

is one. By Lemma 1.6,

$$
\|\mathbf{s}\|_{\infty} \geq \frac{1}{\sqrt{n-r+1}}
$$

We now wish to show that $\left|s_{n-r+1}\right|=\|\mathbf{s}\|_{\infty}$. Since $\widetilde{V} \widetilde{V}^{\mathrm{T}}=\widehat{V} Q Q^{\mathrm{T}} \widehat{V}^{\mathrm{T}}=\widehat{V} \widehat{V}^{\mathrm{T}}$, the determinant of each $r$-by-r submatrix of $\widetilde{V}$ is equal to the determinant of the corresponding $r$-by- $r$ submatrix of $\widehat{V}$. Consequently, $\left|\operatorname{det}\left(\widetilde{V}_{[2]}\right)\right|=$ $\max _{1 \leq i \leq p}\left|\operatorname{det}\left(\widetilde{V}_{i}\right)\right|$, where $\mathscr{L}(\widetilde{V})=\left\{\widetilde{V}_{1}, \ldots, \widetilde{V}_{p}\right\}$ is the set of all $r$-by- $r$ submatrices of $\widetilde{V}$. Notice that $\left|\operatorname{det} \widetilde{V}_{[2]}\right|=\left|s_{n-r+1}\right| \cdot\left|\operatorname{det} \widetilde{V}_{[2]}\left(1^{\prime}\right)\right|$. If $\left|s_{n-r+1}\right| \neq\|\mathbf{s}\|_{\infty}$, there must exist $s_{i} \quad(1 \leq i<n-r+1)$ such that $\left|s_{i}\right| \cdot\left|\operatorname{det} \widetilde{V}_{[2]}\left(1^{\prime}\right)\right|>\left|\operatorname{det} \widetilde{V}_{[2]}\right|$; this is a contradiction.

Now we proceed to prove the inequality $(2.1)$. Let $G=\left(\widehat{\Pi}_{2} V\right)\left(\widehat{\Pi}_{2} V\right)^{\mathrm{T}}=$ $\widehat{\Pi}_{2} V V^{\mathrm{T}} \widehat{\Pi}_{2}^{\mathrm{T}}$ be the $r$-by-r principal submatrix of $\tilde{V} \widetilde{V}^{\mathrm{T}}=\widehat{V} \widehat{V}^{\mathrm{T}}=\widehat{\Pi} V V^{\mathrm{T}} \widehat{\Pi}^{\mathrm{T}}$ at the lower-right corner. It is easy to see that $\operatorname{det}(G)=\left(\operatorname{det}\left(\widehat{\Pi}_{2} V\right)\right)^{2}=s_{n-r+1}^{2}$. $\left(\operatorname{det} \widetilde{V}_{[2]}\left(1^{\prime}\right)\right)^{2}$, and $\operatorname{det}\left(G\left(1^{\prime}\right)\right)=\left(\operatorname{det} \widetilde{V}_{[2]}\left(1^{\prime}\right)\right)^{2}$. Thus we have

$$
\frac{\operatorname{det}\left(G\left(1^{\prime}\right)\right)}{\operatorname{det}(G)}=\frac{1}{s_{n-r+1}^{2}} \leq n-r+1 .
$$

Since we can always permute any row of $\widehat{\Pi}_{2} V$ to its first row without changing its determinant, we can replace $G\left(1^{\prime}\right)$ by $G\left(i^{\prime}\right)$ in $(2.2)$, and the lemma is proved.

Theorem 2.2. For any matrix $A \in \mathbb{R}^{m \times n} \quad(m \geq n)$ and any integer $r \quad(0<r<$ $n)$, there exists a permutation matrix $\Pi \in P_{n}$ such that the diagonal blocks of $R=\left(\begin{array}{cc}R_{11} & R_{12} \\ 0 & R_{22}\end{array}\right)$, the upper triangular factor of the $Q R$ factorization of $A \Pi$ with 
$R_{11} \in \mathbb{R}^{r \times r}$, satisfy the following inequalities:

$$
\left\|R_{22}\right\|_{2} \leq \sqrt{r(n-r)+\min (r, n-r)} \sigma_{r+1}(A),
$$

and

$$
\sigma_{\min }\left(R_{11}\right) \geq \frac{1}{\sqrt{r(n-r)+\min (r, n-r)}} \sigma_{r}(A) .
$$

The permutation matrix $\Pi$ is obtained by selecting an $(n-r)$-by- $(n-r)$ submatrix of $V_{2}$ having the largest determinant in absolute value among all such submatrices of $V_{2}$, and permuting it to the bottom of the matrix $\Pi^{\mathrm{T}} V_{2}$. Here, $U^{\mathrm{T}} A V$ is the SVD of $A$, and $V_{2}$ is formed by the last $(n-r)$ columns of the orthogonal matrix $V$.

Furthermore, if $A$ has numerical rank $r$, then the $Q R$ factorization of $A \Pi$ is guaranteed to be an $R R Q R$ factorization of $A$.

Proof. First, assume that $\Pi \in P_{n}$ is an arbitrary permutation such that $A \Pi=$ $Q R$ is the $\mathrm{QR}$ factorization of $A \Pi$.

Let

$$
\Pi^{\mathrm{T}} V=\left[\begin{array}{l}
\Pi_{1}^{\mathrm{T}} \\
\Pi_{2}^{\mathrm{T}}
\end{array}\right]\left[V_{1} V_{2}\right]=\left[\begin{array}{ll}
\Pi_{1}^{\mathrm{T}} V_{1} & \Pi_{1}^{\mathrm{T}} V_{2} \\
\Pi_{2}^{\mathrm{T}} V_{1} & \Pi_{2}^{\mathrm{T}} V_{2}
\end{array}\right]=: W=\left[\begin{array}{ll}
W_{11} & W_{12} \\
W_{21} & W_{22}
\end{array}\right],
$$

where $W_{22} \in \mathbb{R}^{(n-r) \times(n-r)}$.

According to the C-S decomposition theorem [11, p. 77],

$$
\sigma_{\min }\left(W_{11}\right)=\sigma_{\min }\left(W_{22}\right)=: \rho .
$$

We now wish to determine a lower bound of $\rho$ when we choose $\Pi$ such that

$$
\left|\operatorname{det}\left(\Pi_{2}^{\mathrm{T}} V_{2}\right)\right|=\max _{V_{2}^{\prime} \in \mathscr{L}\left(V_{2}\right)}\left|\operatorname{det}\left(V_{2}^{\prime}\right)\right| .
$$

Notice that $\rho \neq 0$ in (2.6) for the choice of $\Pi$ in (2.7). Now, by well-known adjoint matrix formulas $[13$, p. 20],

$$
\left\|W_{22}^{-1}\right\|_{2}^{2} \leq\left\|W_{22}^{-1}\right\|_{F}^{2}=\sum_{i=1}^{n-r}\left(W_{22}^{-\mathrm{T}} W_{22}^{-1}\right)_{i i}=\sum_{i=1}^{n-r} \frac{\operatorname{det} G\left(i^{\prime}\right)}{\operatorname{det} G},
$$

where $G=W_{22} W_{22}^{\mathrm{T}}$. From Lemma 2.1, with $\Pi$ as chosen in (2.7), we have

$$
\sum_{i=1}^{n-r} \frac{\operatorname{det} G\left(i^{\prime}\right)}{\operatorname{det} G} \leq(n-r) \frac{\max _{1 \leq i \leq n-r} \operatorname{det} G\left(i^{\prime}\right)}{\operatorname{det} G} \leq(n-r)(r+1) .
$$

Having thus proved

$$
\rho \geq \frac{1}{\sqrt{(n-r)(r+1)}}
$$

we easily conclude that

$$
\rho \geq \frac{1}{\sqrt{r(n-r+1)}}
$$

is also true for the same $\Pi$ chosen in (2.7), since by the C-S decomposition theorem we have

$$
\left|\operatorname{det} W_{22}\right|=\left|\operatorname{det} W_{11}\right|=\max _{V_{1}^{\prime} \in \mathscr{L}\left(V_{2}\right)}\left|\operatorname{det}\left(V_{1}^{\prime}\right)\right| .
$$


Thus, inequalities (2.3) and (2.4) are proved by applying Theorem 1.5. The final conclusion is immediate from Definitions 1.1 and 1.3.

The proof of Theorem 2.2 makes it clear that the permutation $\Pi$ which guarantees an RRQR factorization of $A$ can also be obtained by selecting an $r$-by-r submatrix of $V_{1}$ having the largest determinant in absolute value among all such submatrices of $V_{1}$, and by permuting it to the top of the matrix $\Pi^{\mathrm{T}} V_{1}$, where the columns of $V_{1}$ are the right singular vectors of $A$ corresponding to singular values $\sigma_{1}, \ldots, \sigma_{r}$. Moreover, according to the C-S decomposition theorem, the permutations, obtained by inspecting the values of determinants of submatrices of $V_{1}$ or $V_{2}$ in this way are the same.

In Theorem 2.2, we have established the following lower bound for the function $\chi(n, r)$ in general:

$$
\chi(n, r)=\chi(n, n-r) \geq \frac{1}{\sqrt{r(n-r)+\min (r, n-r)}}
$$

This bound is sharp in the sense that when $r=n-1$ or $r=1$ the bound is the exact value of $\chi(n, 1)=1 / \sqrt{n}$; that is, Corollary 1.7 is a special case of this theorem. While there is still room for improvement in other cases, the bound is sharp enough for our purpose.

It is interesting to note the relation between the smallest singular value and the absolute value of the determinant of an $r$-by- $r$ submatrix of a matrix with $r$ orthonormal columns. In Theorem 2.2 we actually proved that, when a submatrix of an orthogonal matrix has maximum determinant in absolute value, its smallest singular value must be relatively large. In $\S 3$ we present some computational results illustrating this fact.

Finally, we point out that the columns of the matrix $V_{2}$ may be replaced by any orthonormal basis of the numerical null space of $A$ without invalidating Theorem 2.2 (see Remark 3).

As we mentioned in $\S 2$, an RRQR factorization of $A$ can be defined using other matrix norms. The following existence theorem for RRQR involves the Frobenius norm.

Theorem 2.3. For any matrix $A \in \mathbb{R}^{m \times n}(m \geq n)$ and any integer $r \quad(0<$ $r<n)$ for which $\sigma_{r}(A) \neq 0$, there exists a permutation matrix $\Pi$ such that the diagonal blocks of $R=\left(\begin{array}{cc}R_{11} & R_{12} \\ 0 & R_{22}\end{array}\right)$, the upper triangular factor of the $Q R$ factorization of $A \Pi$ with $R_{11} \in \mathbb{R}^{r \times r}$, satisfy the following inequalities:

$$
\begin{gathered}
\left\|R_{22}\right\|_{F} \leq \sqrt{(n-r)(r+1)} \sigma_{r+1}(A), \\
\left\|R_{11}^{-1}\right\|_{F} \leq \sqrt{(r(n-r+1))} / \sigma_{r}(A) .
\end{gathered}
$$

Proof. First, assume that $\Pi \in P_{n}$ is an arbitrary permutation such that $A \Pi=$ $Q R$. Let $V$ be the orthogonal matrix such that

$$
V^{\mathrm{T}} A^{\mathrm{T}} A V=\left[\begin{array}{ccc}
\sigma_{1}^{2} & & \\
& \ddots & \\
& & \sigma_{n}^{2}
\end{array}\right]=\Sigma^{2},
$$


where the $\sigma_{i}$ are the singular values of $A$ with $\sigma_{1} \geq \sigma_{2} \geq \cdots \geq \sigma_{n}$. From

$$
\begin{aligned}
\Sigma^{2}= & V^{\mathrm{T}} \Pi R^{\mathrm{T}} R \Pi^{\mathrm{T}} V \\
= & V^{\mathrm{T}} \Pi\left(\begin{array}{cc}
R_{11}^{\mathrm{T}} & 0 \\
R_{12}^{\mathrm{T}} & 0
\end{array}\right)\left(\begin{array}{cc}
R_{11} & R_{12} \\
0 & 0
\end{array}\right) \Pi^{\mathrm{T}} V \\
& +V^{\mathrm{T}} \Pi\left(\begin{array}{cc}
0 & 0 \\
0 & R_{22}^{\mathrm{T}}
\end{array}\right)\left(\begin{array}{cc}
0 & 0 \\
0 & R_{22}
\end{array}\right) \Pi^{\mathrm{T}} V,
\end{aligned}
$$

we see that

$$
\Sigma^{2}-V^{\mathrm{T}} \Pi\left(\begin{array}{cc}
0 & 0 \\
0 & R_{22}^{\mathrm{T}}
\end{array}\right)\left(\begin{array}{cc}
0 & 0 \\
0 & R_{22}
\end{array}\right) \Pi^{\mathrm{T}} V \succeq 0
$$

Thus,

$$
\left[\begin{array}{ccc}
\sigma_{r+1}^{2} & & \\
& \ddots & \\
& & \sigma_{n}^{2}
\end{array}\right]-W_{22}^{\mathrm{T}} R_{22}^{\mathrm{T}} R_{22} W_{22} \succeq 0,
$$

where

$$
\begin{aligned}
\Pi^{\mathrm{T}} V & =\left[\begin{array}{l}
\Pi_{1}^{\mathrm{T}} \\
\Pi_{2}^{\mathrm{T}}
\end{array}\right]\left[V_{1} V_{2}\right]=\left[\begin{array}{ll}
\Pi_{1}^{\mathrm{T}} V_{1} & \Pi_{1}^{\mathrm{T}} V_{2} \\
\Pi_{2}^{\mathrm{T}} V_{1} & \Pi_{2}^{\mathrm{T}} V_{2}
\end{array}\right] \\
& =: W=\left[\begin{array}{ll}
W_{11} & W_{12} \\
W_{21} & W_{22}
\end{array}\right]
\end{aligned}
$$

with $W_{11} \in \mathbb{R}^{r \times r}$.

Now (2.15) can be written as

$$
\sigma_{r+1}^{2} W_{22}^{-\mathrm{T}} W_{22}^{-1}-R_{22}^{\mathrm{T}} R_{22} \succeq 0 .
$$

Here the invertibility of $\Pi_{2}^{\mathrm{T}} V_{2}=W_{22}$ is guaranteed by our choice of $\Pi$ later in (2.17). Since the diagonal of a positive semidefinite matrix is nonnegative, we have

$$
\begin{aligned}
\left(R_{22}^{\mathrm{T}} R_{22}\right)_{i i} & \leq\left(\left(W_{22} W_{22}^{\mathrm{T}}\right)^{-1}\right)_{i i} \cdot \sigma_{r+1}^{2}(A) \\
& =\frac{\operatorname{det}\left(G\left(i^{\prime}\right)\right)}{\operatorname{det}(G)} \sigma_{r+1}^{2}(A), \quad i=1, \ldots, n-r,
\end{aligned}
$$

where $G=W_{22} W_{22}^{\mathrm{T}}$. The equality above is obtained by invoking well-known adjoint matrix formulas $[13$, p. 20$]$.

It follows immediately that

$$
\begin{aligned}
\left\|R_{22}\right\|_{F}^{2} & \leq(n-r) \max _{1 \leq i \leq n-r}\left(R_{22}^{\mathrm{T}} R_{22}\right)_{i i} \\
& \leq(n-r) \cdot \frac{\max _{1 \leq i \leq n-r}\left(\operatorname{det}\left(G\left(i^{\prime}\right)\right)\right)}{\operatorname{det}(G)} \sigma_{r+1}^{2}(A) .
\end{aligned}
$$

Applying Lemma 2.1, we know that we can always choose $\widehat{\Pi} \in P_{n}$ such that

$$
\operatorname{det}\left(\widehat{\Pi}_{2}^{\mathrm{T}} V_{2}\right)=: \operatorname{det}\left(\widehat{W}_{22}\right)=\max _{\Pi \in P_{n}}\left(\Pi_{2}^{\mathrm{T}} V_{2}\right)
$$

to make

$$
\frac{\max _{1 \leq i \leq n-r}\left(\operatorname{det}\left(G\left(i^{\prime}\right)\right)\right)}{\operatorname{det}(G)} \leq(r+1) .
$$

Hence, (2.12) is proved. 
On the other hand, from (2.14) we have

$$
\Pi^{\mathrm{T}} V \Sigma^{2} V^{\mathrm{T}} \Pi=\left(\begin{array}{cc}
R_{11}^{\mathrm{T}} R_{11} & * \\
* & *
\end{array}\right) .
$$

If we partition $\Pi^{\mathrm{T}} V$ as in (2.16), it follows that

$$
W_{11}\left(\begin{array}{ccc}
\sigma_{1}^{2} & & \\
& \ddots & \\
& & \sigma_{r}^{2}
\end{array}\right) W_{11}^{\mathrm{T}}+W_{12}\left(\begin{array}{ccc}
\sigma_{r+1}^{2} & & \\
& \ddots & \\
& & \sigma_{n}^{2}
\end{array}\right) W_{12}^{\mathrm{T}}=R_{11}^{\mathrm{T}} R_{11} .
$$

Consequently,

$$
R_{11}^{\mathrm{T}} R_{11}-W_{11}\left(\begin{array}{ccc}
\sigma_{1}^{2} & & \\
& \ddots & \\
& & \sigma_{r}^{2}
\end{array}\right) W_{11}^{\mathrm{T}} \succeq 0
$$

and, in particular,

$$
R_{11}^{\mathrm{T}} R_{11}-\sigma_{r}^{2}\left(W_{11} W_{11}^{\mathrm{T}}\right) \succeq 0 .
$$

Therefore, $R_{11}$ is nonsingular, since $\sigma_{r} \neq 0$. It follows [13, p. 471] that

$$
\frac{1}{\sigma_{r}^{2}} W_{11}^{-\mathrm{T}} W_{11}^{\mathrm{T}}-R_{11}^{-1} R_{11}^{-\mathrm{T}} \succeq 0
$$

Here again, our final choice of $\Pi$ in (2.19) guarantees $\Pi_{1}^{\mathrm{T}} V_{1}$ to be invertible. Using Lemma 2.1 and arguments similar to those used to prove (2.12), one can also prove that

$$
\left\|R_{11}^{-1}\right\|_{F}^{2} \leq r \cdot \frac{\max _{1 \leq i \leq r}\left(\operatorname{det}\left(H\left(i^{\prime}\right)\right)\right)}{\operatorname{det}(H)} \cdot \frac{1}{\sigma_{r}^{2}(A)},
$$

where $H=W_{11} W_{11}^{\mathrm{T}}$.

Applying the C-S decomposition theorem to (2.18), we can easily show that $\operatorname{det}\left(W_{11}\right)=\operatorname{det}\left(\Pi_{1}^{\mathrm{T}} V_{1}\right)=\operatorname{det}\left(\Pi_{2}^{\mathrm{T}} V_{2}\right)=\operatorname{det}\left(W_{22}\right)$ for any $\Pi \in P_{n}$. Thus, when we choose a particular $\widehat{\Pi} \in P_{n}$ such that $\left|\operatorname{det}\left(\widehat{\Pi}_{2}^{\mathrm{T}} V_{2}\right)\right|=\max _{\Pi \in P_{n}}\left|\operatorname{det}\left(\Pi_{2} V_{2}\right)\right|$, as was done in proving the inequality (2.12), the same permutation matrix $\widehat{\Pi}$ will also guarantee that

$$
\operatorname{det}\left(\widehat{\Pi}_{1} V_{1}\right)=\max _{\Pi \in P_{n}}\left|\operatorname{det}\left(\Pi_{1} V_{1}\right)\right|
$$

By Lemma 2.1,

$$
\max _{1 \leq i \leq r} \frac{\operatorname{det} H\left(i^{\prime}\right)}{\operatorname{det} H} \leq n-r+1 .
$$

Substitution of (2.20) into (2.18) then proves the inequality (2.13).

\section{NUMERICAL EXAMPLES}

In this section, we provide some examples to illustrate our existence theorem by exhibiting the relations between the various key values we have discussed so far. All numerical computations were done on a Sun-3/80, using MATLAB. We present two examples: Kahan's example, and a rather general random matrix. For each example, we construct a matrix with known numerical rank $r$ and known right singular vectors corresponding to singular values $\sigma_{r+1}, \ldots, \sigma_{n}$ 
which are near zero. According to Remark 1.2, there is a total of $C(n, r)$ different permutations we must consider. On the horizontal coordinate axis of Figures 1 and 2, each positive integer represents a permutation. For each permutation $\Pi$, we exhibit four different values: $\left\|R_{22}\right\|_{2}, \sigma_{\min }\left(R_{11}\right),\left|\operatorname{det}\left(\Pi_{2}^{\mathrm{T}} V_{2}\right)\right|$, and $\sigma_{\min }\left(\Pi_{2}^{\mathrm{T}} V_{2}\right)$ (see (2.3), (2.4), and (2.5)). These four values will be considered as functions of the permutations (represented by integers), and they are represented by four different curves in the figures. To visualize the relationship between these four values, we order the permutations so that the value of $\left|\operatorname{det}\left(\Pi_{2}^{\mathrm{T}} V_{2}\right)\right|$ is a monotonically nondecreasing function of the permutations. For illustration, the "machine" precision is $\mu=0.0001$.

Example 3.1. Let $K_{n}(c) \in \mathbb{R}^{n \times n}$ be an upper triangular matrix defined by

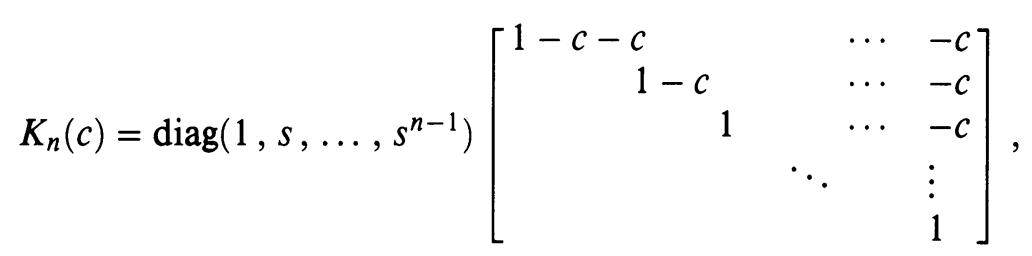

where $c^{2}+s^{2}=1$.

This is a counterexample given by Kahan [14], which shows that the QR factorization algorithm with column pivoting strategy does not always produce an RRQR. In Figure 1, we choose $A=K_{50}(0.2)$ to illustrate our theorem. From computation we have $\sigma_{50}(A)=0.000091$ and $\sigma_{49}(A)=0.41124$. It is clear that the numerical rank of $A$ should be 49 if $\mu=0.0001$. Now we consider the $\mathrm{QR}$ factorization of $A \Pi$, where $\Pi \in P_{50}$. As indicated in Remark 1.2, we need only to consider 50 different permutations in order to get the possible 50 different pairs of values of $\sigma_{\min }\left(R_{11}\right)$ (curve (A)) and $\left\|R_{22}\right\|_{2}$ (curve (B)). We order these permutations such that the value of $\left|\operatorname{det}\left(\Pi_{2}^{\mathrm{T}} \mathbf{v}_{n}\right)\right|=\sigma_{\min }\left(\Pi_{2}^{\mathrm{T}} \mathbf{v}_{n}\right)=$ $\left|\left(\Pi^{\mathrm{T}} \mathbf{v}_{n}\right)\right|$ (curve (C)), where $\mathbf{v}_{n}$ is the right singular vector corresponding to the smallest singular value of $A$, is a monotonically increasing function of the permutations, when the permutations are numbered by the positive integers on the horizontal axis. With the permutations so arranged, it turns out that the integer $i$ on the horizontal axis in Figure 1 represents the permutation that permutes the $(n-i+1)$ st column of $A$ to the last column and keeps all other columns in their original order. For example, from Figure 1 we see that the "most" rank-revealing $\mathrm{QR}$ of $A$ is obtained by permuting the first column of $A$ to the last column and moving the remaining columns one column to the left. In this case, $\left\|R_{22}\right\|_{2}=\left|r_{50,50}\right|=0.00017$, and $\sigma_{\min }\left(R_{11}\right)=0.41124$ (the corresponding theoretical bounds in Theorem 2.2 are 0.00064 and 0.05816 ), and according to our definition we have an RRQR of $A$. For this permutation, the value of $\left|\operatorname{det}\left(\Pi_{2}^{\mathrm{T}} \mathbf{v}_{n}\right)\right|$ reaches its maximum, 0.55277. It is interesting to note that the triangular matrix $A$ itself, represented by 1 on the horizontal axis in Figure 1, is the "most" "non-rank-revealing" case among all the 50 cases, where $\left\|R_{22}\right\|_{2}=\left|r_{50,50}\right|=s^{n-1}=0.36783$ and $\sigma_{\min }\left(R_{11}\right)=0.00011$.

It is also worth noting that all the curves in Figure 1 are monotonic. Thus, when the value of $\left|\operatorname{det}\left(\Pi_{2}^{\mathrm{T}} \mathbf{v}_{n}\right)\right|=\sigma_{\min }\left(\Pi_{2}^{\mathrm{T}} \mathbf{v}_{n}\right)$ (curve (C)) increases, the value of $\sigma_{\min }\left(R_{11}\right)$ (curve $\left.(\mathrm{A})\right)$ also increases, and the value of $\left\|R_{22}\right\|_{2}$ (curve (B)) decreases. Therefore, the corresponding $\mathrm{QR}$ factorization of $A \Pi$ gradually becomes a rank-revealing one. 


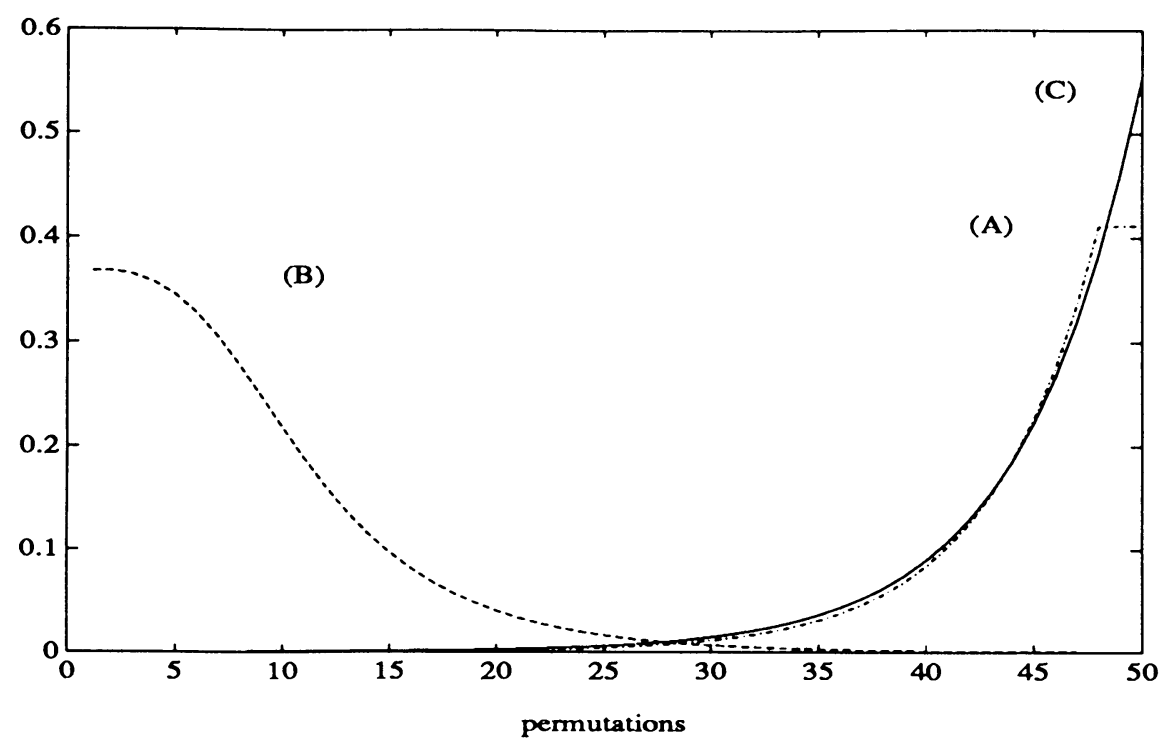

FIGURE 1. Kahan's example

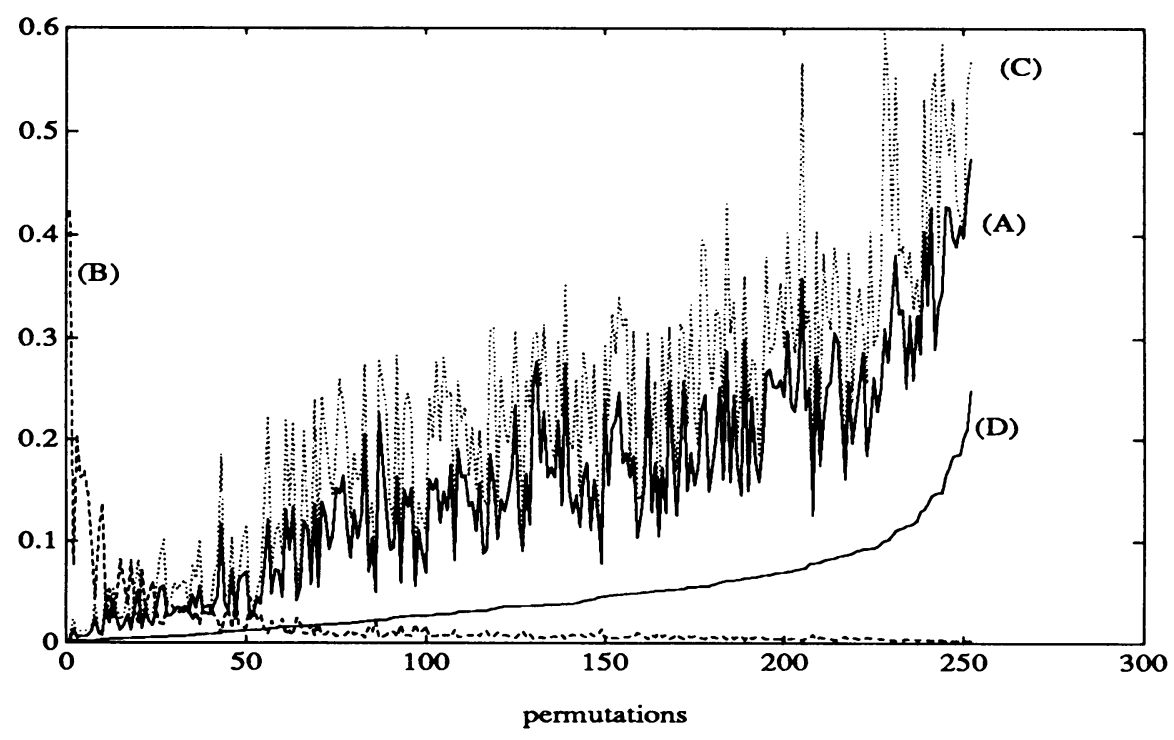

FIGURE 2. Example 3.2

Example 3.2. Let $A \in R^{50 \times 10}$ be given by

$$
A=Q_{50}\left[\begin{array}{c}
\Sigma \\
0
\end{array}\right] Q_{10},
$$

where $\Sigma=\operatorname{diag}(2,2,2,1,1, \mu, \mu, \mu, \mu, \mu)$ with $\mu=0.0001$, and $Q_{n} \in$ $\mathbb{R}^{n \times n} \quad(n=10,50)$ are randomly generated orthogonal matrices. For this example, there is a total of 252 permutations represented by the integers on the horizontal axis in Figure 2. Again, all the permutations $\Pi$ are so ordered that the corresponding value of $\left|\operatorname{det}\left(\Pi_{2}^{\mathrm{T}} V_{2}\right)\right|$ (curve (D)), where the columns of matrix $V_{2}$ are the right singular vectors associated with singular values 
$\sigma_{6}=\cdots=\sigma_{10}=\mu$, is a monotonically nondecreasing function of the permutations represented by integers. Curve (A) in Figure 2 is the value of $\sigma_{\min }\left(\Pi_{2}^{\mathrm{T}} V_{2}\right)$, curve (C) the value of $\sigma_{\min }\left(R_{11}\right)$, and curve (B) the value of $5\left\|R_{22}\right\|_{2}$. In this example, none of the curves is monotonic except curve (D). However, if one ignores the local oscillation, these three curves are "globally" monotonic. Globally, when curve (D) $\left(\left|\operatorname{det}\left(\Pi_{2}^{\mathrm{T}} V_{2}\right)\right|\right)$ increases, curve $(\mathrm{A})\left(\sigma_{\min }\left(\Pi_{2}^{\mathrm{T}} V_{2}\right)\right)$ also increases, and the corresponding QR factorization of $A \Pi$ becomes more rankrevealing as a result of the increasing gap between $\sigma_{\min }\left(R_{11}\right)$ and $\left\|R_{22}\right\|_{2}$. It is interesting to note that when the permutation is chosen so that $\left|\operatorname{det}\left(\Pi_{2}^{\mathrm{T}} V_{2}\right)\right|=$ 0.24714 reaches a maximum, $\sigma_{\min }\left(\Pi_{2}^{\mathrm{T}} V_{2}\right)=0.47453$ also reaches a maximum. (While this result is not always true, we found it to occur very frequently indeed.) For this permutation (represented by 252), $\left\|R_{22}\right\|_{2}=0.00021$ and $\sigma_{\min }\left(R_{11}\right)=0.56699$, while the theoretical bounds in Theorem 2.2 are 0.00054 and 0.18257 , respectively.

\section{CONCLUDING REMARKS}

(1) In this paper, we have proved the following theorem:

Theorem 4.1. A matrix $A \in \mathbb{R}^{m \times n} \quad(m \geq n)$ has numerical rank $r$ if and only if there is a permutation $\Pi \in P_{n}$ such that $A \Pi=Q R$ is an $R R Q R$ factorization of $A$.

Moreover, the permutation $\Pi$ can be obtained by inspecting the determinants of certain submatrices in $V$, whose columns are the right singular vectors of $A$. That is, if any orthonormal basis of the numerical null space of a matrix $A$ is known, Theorem 2.2 suggests a completely reliable algorithm to find an $\mathrm{RRQR}$ factorization of $A$. Because it is costly, however, this algorithm has more theoretical than practical value.

(2) It is clear that Theorem 2.2 also provides a completely reliable algorithm for solving the so-called "subset selection" or "column selection" problem [11, p. 571;10,20]. In comparison with the algorithm recommended in [11], our algorithm is not too expensive, since the first phase of both algorithms is to find the SVD (or at least the partial SVD [21]).

(3) Theorem 2.2 essentially provides the estimation of the largest possible gap between the corresponding values of $\sigma_{\min }\left(R_{11}\right)$ and $\left\|R_{22}\right\|_{2}$ in terms of a given gap between any two adjacent singular values of a matrix $A$, regardless of whether the latter gap actually means a "numerical rank" or not.

APPendix. Proof of Lemma 1.8

Let $\delta_{i j}$ and $\mu_{i j}$ with $\left(\delta_{i j} \geq \mu_{i j}\right)$ be the squares of the two singular values of $V_{i j}$, the submatrix formed by the $i$ th and $j$ th rows of $V \in \mathscr{O}_{n, 2}$. Let $V V^{\mathrm{T}}=C$. We know that

$$
\operatorname{trace}(C)=2
$$

and that

$$
\sum_{1 \leq i<j \leq n} \operatorname{det}(C(i j))=1
$$


where $C(i j)=\left(\begin{array}{l}c_{i i} \\ c_{j i} c_{j j}\end{array}\right)$ are the principal submatrices of $C$. Clearly,

$$
C(i j)=V_{i j} V_{i j}^{\mathrm{T}} \text {. }
$$

Thus, we have, for each pair $(i, j), 1 \leq i<j \leq n$,

$$
\begin{gathered}
\delta_{i j}+\mu_{i j}=c_{i i}+c_{j j}, \\
\delta_{i j} \cdot \mu_{i j}=\operatorname{det}(C(i j)) .
\end{gathered}
$$

From (A.4), (A.5), and (A.2) we have

$$
\sum_{1 \leq i<j \leq n} \delta_{i j}\left(c_{i i}+c_{j j}-\delta_{i j}\right)=1
$$

and

$$
\sum_{1 \leq i<j \leq n} \mu_{i j}\left(c_{i i}+c_{j j}-\mu_{i j}\right)=1 \text {. }
$$

If we let $x_{i j}$ denote either $\mu_{i j}$ or $\delta_{i j}$ in (A.6) and (A.7), we can rewrite these relations as

$$
\sum_{1 \leq i<j \leq n}\left(x_{i j}-\frac{c_{i i}+c_{j j}}{2}\right)^{2}-\sum_{1 \leq i<j \leq n}\left(\frac{c_{i i}+c_{j j}}{2}\right)^{2}+1=0 .
$$

By using (A.1), (A.2), $C=C^{\mathrm{T}}$, and $C^{2}=C$, it is easy to show that

$$
\begin{aligned}
\sum_{1 \leq i<j \leq n}\left(c_{i i}+c_{j j}\right)^{2} & =\sum_{1 \leq i<j \leq n}\left(c_{i i}^{2}+c_{j j}^{2}\right)+2 \sum_{1 \leq i<j \leq n} c_{i i} c_{j j} \\
& =(n-1) \sum_{i=1}^{n} c_{i i}^{2}+2\left(1+\sum_{1 \leq i<j \leq n} c_{i j}^{2}\right) \\
& =(n-1) \sum_{i=1}^{n} c_{i i}^{2}+2-\sum_{i=1}^{n} c_{i i}^{2}+2 \\
& =(n-2) \sum_{i=1}^{n} c_{i i}^{2}+4 .
\end{aligned}
$$

Applying (A.9), we can rewrite equation (A.8) as

$$
\sum_{1 \leq i<j \leq n}\left(x_{i j}-\frac{c_{i i}+c_{j j}}{2}\right)^{2}=\left(\frac{n-2}{4}\right) \sum_{i=1}^{n} c_{i i}^{2} .
$$

Define vectors $\mathbf{a}, \mathbf{x} \in \mathbb{R}^{C(n, 2)}$ by

$$
\mathbf{a}=\left(\frac{c_{11}+c_{22}}{2}, \frac{c_{11}+c_{33}}{2}, \ldots, \frac{c_{n-1, n-1}+c_{n, n}}{2}\right)
$$

and

$$
\mathbf{x}=\left(x_{11}, x_{12}, \ldots, x_{n-1, n}\right),
$$

where the double subscripts are lexicographically ordered. Then (A.10) can be written as

$$
\|\mathbf{x}-\mathbf{a}\|_{2}=r, \quad r=\frac{\sqrt{n-2}}{2} \sqrt{\sum_{i=1}^{n} c_{i i}^{2}} .
$$


Consider now the following optimization problem:

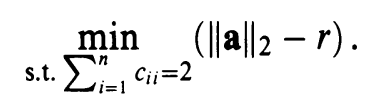

Applying the Lagrange multiplier method, we have (for fixed $i$ )

$$
\begin{aligned}
\frac{\partial f}{\partial c_{i i}}= & \frac{1}{2} \frac{1}{\sqrt{\sum\left(\left(c_{i i}+c_{j j}\right) / 2\right)^{2}}} \frac{1}{2} \sum_{i \neq j}\left(c_{i i}+c_{j j}\right) \\
& -\frac{\sqrt{n-2}}{2} \cdot \frac{1}{2} \cdot \frac{1}{\sqrt{\sum_{i=1}^{n} c_{i i}^{2}}} \cdot 2 c_{i i}=\lambda,
\end{aligned}
$$

or $[(n-2) p-q] c_{i i}=\lambda-2 p$ for each $i$, where

$$
\frac{1}{p}=4 \sqrt{\sum\left(\frac{c_{i i}+c_{i j}}{2}\right)^{2}}, \quad q=\frac{\sqrt{n-2}}{2 \sqrt{\sum_{i=1}^{n} c_{i i}^{2}}} .
$$

From (A.9) we know that $(n-2) p-q \neq 0$, and (A.13) attains its minimum at $c_{i i}=2 / n, i=1, \ldots, n$. The maximum value of (A.13) is

$$
\sqrt{\sum_{1 \leq i<j \leq n}\left(\frac{2}{n}\right)^{2}}-\frac{\sqrt{n-2}}{2} \sqrt{\sum_{1}^{n}\left(\frac{2}{n}\right)^{2}}=\frac{\sqrt{n}}{\sqrt{2 n-2}+\sqrt{n-2}} .
$$

However, we have

$$
\|\mathbf{x}\|_{2} \geq\|\mathbf{a}\|_{2}-\|\mathbf{x}-\mathbf{a}\|_{2}=\|\mathbf{a}\|_{2}-r \geq \frac{\sqrt{n}}{\sqrt{2 n-2}+\sqrt{n-2}},
$$

and consequently

$$
\|\mathbf{x}\|_{\infty} \geq 2 \sqrt{\frac{2}{n(n-1)}} \cdot\|\mathbf{x}\|_{2} \geq \frac{1}{n}\left(2-\sqrt{2} \sqrt{\frac{n-2}{n-1}}\right)
$$

for any $V \in \mathscr{O}_{n, 2}$ such that $\|\mathbf{x}-\mathbf{a}\|_{2}=r$. Thus,

$$
\min \|\mathbf{x}\|_{\infty} \geq \frac{1}{n}\left(2-\sqrt{2} \sqrt{\frac{n-2}{n-1}}\right)
$$

for any $V \in \mathscr{O}_{n, 2}$ such that $\|\mathbf{x}-\mathbf{a}\|_{2}=r$. In particular, when $x_{i j}=\mu_{i j}=$ $\sigma_{\min }\left(V_{i j}\right),(\mathrm{A} .14)$ is true.

\section{ACKNOWLEDGMENTS}

The authors are grateful to many of our colleagues, who have taken time to read our original manuscript, for their valuable comments and suggestions. Special thanks are due to Professors Walter Gautschi, Per Christian Hansen, and Beresford Parlett for their detailed suggestions for improving the presentation. The second author would also like to thank Chris Bischof and Peter Tang for their stimulating discussions on related subjects during his visit to the Mathematics and Computer Science Division at Argonne National Laboratory. 


\section{BIBLIOGRAPHY}

1. N. Anderson and I. Karasalo, On computing bounds for the least singular value of a triangular matrix, BIT 15 (1975), 1-4.

2. C. H. Bischof and P. C. Hansen, Structure-preserving and rank-revealing $Q R$ factorizations, Report MCS-P100-0989, Mathematics and Computer Science Division, Argonne National Laboratory, 1989. (To appear in SIAM J. Sci. Statist. Comput.)

3. P. Businger and G. H. Golub, Linear least squares solutions by Householder transformations, Numer. Math. 1 (1965), 269-276.

4. T. F. Chan, Deflated decomposition of solutions of nearly singular systems, SIAM J. Numer. Anal. 21 (1984), 738-754.

5. __ Alternative to the SVD: rank revealing QR-factorizations, Advanced Algorithms and Architectures for Signal Processing (J. M. Speiser, ed.), SPIE Proceedings, vol. 696, 1986.

6. __ Rank revealing QR factorizations, Linear Algebra Appl. 88/89 (1987), 67-82.

7. T. F. Chan and P. C. Hansen, Computing truncated singular value decomposition least squares solutions by rank revealing QR-factorizations, SIAM J. Sci. Statist. Comput. 11 (1990), 519-530.

8. L. Foster, Rank and null space calculations using matrix decomposition without column interchanges, Linear Algebra Appl. 74 (1986), 47-71.

9. G. H. Golub, Numerical methods for solving least squares problems, Numer. Math. 7 (1965), 206-216.

10. G. H. Golub, V. Klema, and G. W. Stewart, Rank degeneracy and least squares problems, Report TR-456, Dept. of Computer Science, Univ. of Maryland, 1976.

11. G. H. Golub and C. F. Van Loan, Matrix computations, 2nd ed., John Hopkins Univ. Press, 1989.

12. R. J. Hanson and C. L. Lawson, Solving least squares problems, Prentice-Hall, 1974.

13. R. A. Horn and C. A. Johnson, Matrix analysis, Cambridge Univ. Press, 1985.

14. W. Kahan, Numerical linear algebra, Canad. Math. Bull. 9 (1966), 757-801.

15. I. Karasalo, $A$ criterion for truncation of the $Q R$-decomposition algorithm for the singular linear least squares problem, BIT 14 (1974), 156-166.

16. T. Manteuffel, An interval analysis approach to rank determination in linear least squares problems, SIAM J. Sci. Statist. Comput. 2 (1981), 335-348.

17. $\mathrm{E} . \mathrm{Ng}$, A scheme for handling rank deficiency in the solution of sparse linear least squares problems, Report ORNL/TM-10980, Mathematics Division, Oak Ridge National Laboratory, 1988.

18. G. W. Stewart, On the implicit deflation of nearly singular systems of linear equations, SIAM J. Sci. Statist. Comput. 2 (1981), 136-140.

19. __ Rank degeneracy, SIAM J. Sci. Statist. Comput. 5 (1984), 403-413.

20. __ Incremental condition calculation and column selection, Report CS-TR-2495, Dept. of Computer Science, Univ. of Maryland, 1990.

21. S. Van Huffel, J. Vandewalle, and A. Haegemans, An efficient and reliable algorithm for computing the singular subspace of a matrix, associated with its smallest singular values, J. Comput. Appl. Math. 19 (1987), 313-330.

Department of Mathematical Sciences, Northern Illinois University, DeKalb, IlliNOIS 60115-2888

E-mail address, C.-T. Pan: pan@math.niu.edu 